\title{
Seismic Response of a Two-Degree-of-Freedom System with Friction Based on the Mass Ratio*
}

\author{
Akinori TOMODA $^{* *}$, Tetsuya WATANABE ${ }^{* *}$ and Kihachiro TANAKA ${ }^{* *}$ \\ **Division of Mechanical Engineering and Science, Saitama University, \\ 255 Shimo-Okubo, Sakura-ku, Saitama 338-8570, Japan \\ E-mail: s08dh002@mail.saitama-u.ac.jp
}

\begin{abstract}
The friction force is effective for dissipating seismic energy. Therefore, in recent years, the installation of a frictional isolator in industrial facilities has been investigated. When a system including this isolator is regarded as multi-dof system, however, the seismic response of the system is obtained only by non-linear time history analysis. A great deal of time is required for non-linear analysis. In the present study, the easy estimation of seismic response for a multi-dof system with friction is considered. The seismic response of a multi-dof linear system can be obtained by the response spectrums method. This estimation is based on the modal analysis method. In the present paper, a method of modal separation for a 2-dof system with friction is presented. However, the accuracy of the proposed method depends on the mass ratio $\gamma$, the frequency ratio $R_{f}$ and the friction force. The proposed method is compared with 2-dof non-linear time history analysis, and the accuracy of the proposed method is calculated at $\gamma \leqq 0.1$ and $R_{f} \leqq 3$. The present study defines the area within an error of $10 \%$ as the applicable area of the modal analysis method for a friction system and shows the range of error over $10 \%$. The proposed method can be useful for obtaining the seismic response of a 2-dof friction system without non-linear time history analysis.
\end{abstract}

Key words: Seismic Design, Modal Separation, Mass Ratio, Friction Force, Structure, Support

\section{Introduction}

Recently, massive earthquakes such as the Niigata-ken Chuetsu-oki earthquake (2007) or the Tokachi-oki earthquake (2003) have occurred frequently in Japan. Large-magnitude earthquakes seriously damage not only ordinary houses, but also structures in industrial facilities. In order to prevent hazardous material spills and secondary disasters, industrial facilities are required to have high aseismic performance compared with houses.

On the other hand, the frictional isolator has attracted attention for the reduction of seismic response in industrial facilities. This system has the effects of shifting the natural frequency away from the predominant frequency of the seismic wave and dissipating seismic energy. These effects can improve the aseismic capacity of a structure in industrial facilities and reduce the cost of seismic design. When a structure is a multi-dof system with friction, however, the seismic response of this system can be obtained by only non-linear time history analysis ${ }^{(1)}$. A great deal of time is necessary for non-linear analysis. In general, immediate and easy estimation of the seismic response is required in the seismic design of industrial facilities. In the previous studies ${ }^{(2)-(6)}$, the authors proposed a response spectrum for a 1-dof system with friction. This spectrum can simply estimate the seismic response of a 1-dof system with friction. 
The present study deals with the easy estimation of seismic response for a multi-dof system with friction using the response spectrums method. The 1-dof linear system and the 1-dof friction system are used in this method. This estimation is based on the modal analysis $\operatorname{method}^{(7)}$. The authors also proposed a method of modal separation to use one slip direction for a piping system with friction ${ }^{(8)}$. In addition, Hanawa et al. proposed a method that is available for any slip direction ${ }^{(9)}$. When we consider the simple 2-dof system with friction, however, the method of modal separation for same directional friction force has not yet been proposed.

In the present paper, the method of modal separation for a 2-dof system with friction is proposed when the mass ratio of the structure and the support is nearly zero. The 1-dof system, which is converted to modal space using this method, calculates the time history response. The time history response on modal space is then translated to real coordinates. An application of the proposed method is discussed by comparing the results of modal analysis for a friction system with the 2-dof non-linear time history analysis.

\section{Modal separation of the 2-dof system with friction}

Figure 1 shows the 2-dof system with friction. The equation of this system is as follows:

$$
\left[\begin{array}{cc}
m_{c} & 0 \\
0 & m_{p}
\end{array}\right]\left\{\begin{array}{l}
\ddot{x}_{c} \\
\ddot{x}_{p}
\end{array}\right\}+\left[\begin{array}{cc}
c_{c} & -c_{c} \\
-c_{c} & c_{c}+c_{p}
\end{array}\right]\left\{\begin{array}{l}
\dot{x}_{c}-\dot{y} \\
\dot{x}_{p}-\dot{y}
\end{array}\right\}+\left[\begin{array}{cc}
k_{c} & -k_{c} \\
-k_{c} & k_{c}+k_{p}
\end{array}\right]\left\{\begin{array}{l}
x_{c}-y \\
x_{p}-y
\end{array}\right\}+\left\{\begin{array}{l}
0 \\
f \operatorname{sign}\left(\dot{x}_{p}-\dot{y}\right)
\end{array}\right\}=0
$$

where $m_{c}, m_{p}, c_{c}, c_{p}, k_{c}, k_{p}$ and $f$ are the mass of the structure, the mass of the support, the damping coefficient of the structure, the damping coefficient of the support, the spring constant of the structure, the spring constant of the support, and the friction force between the support and the base, respectively. In addition, $\operatorname{sign}(x)$ is the signum function and is defined as follows:

$$
\operatorname{sign}(x)= \begin{cases}-1 & (x<0) \\ 0 & (x=0) \\ 1 & (x>1)\end{cases}
$$

Using the relative displacement between structure and base $z_{c}\left(=x_{c}-y\right)$ and the relative displacement between support and base $z_{p}\left(=x_{p}-y\right)$, equation (1) can be written in the following form:

$$
\left[\begin{array}{cc}
m_{c} & 0 \\
0 & m_{p}
\end{array}\right]\left\{\begin{array}{l}
\ddot{z}_{c} \\
\ddot{z}_{p}
\end{array}\right\}+\left[\begin{array}{cc}
c_{c} & -c_{c} \\
-c_{c} & c_{c}+c_{p}
\end{array}\right]\left\{\begin{array}{l}
\dot{z}_{c} \\
\dot{z}_{p}
\end{array}\right\}+\left[\begin{array}{cc}
k_{c} & -k_{c} \\
-k_{c} & k_{c}+k_{p}
\end{array}\right]\left\{\begin{array}{l}
z_{c} \\
z_{p}
\end{array}\right\}=-\left\{\begin{array}{l}
m_{c} \\
m_{p}
\end{array}\right\} \ddot{y}-\left\{\begin{array}{l}
0 \\
f \operatorname{sign}\left(\dot{z}_{p}\right)
\end{array}\right\}
$$

Next, the modal matrix of this system is obtained by equation (1) without the friction force and damping ${ }^{(10)}$. The modal matrix is expressed as follows:

$$
[\phi]=\left[\begin{array}{ll}
\boldsymbol{\varphi}_{1} & \boldsymbol{\varphi}_{2}
\end{array}\right]=\left[\begin{array}{ll}
\phi_{11} & \phi_{12} \\
\phi_{21} & \phi_{22}
\end{array}\right]=\left[\begin{array}{cc}
-f_{c}^{2} & -f_{c}^{2} \\
f_{1}^{2}-f_{c}^{2} & f_{2}^{2}-f_{c}^{2}
\end{array}\right]
$$

where $f_{c}, f_{p}, f_{1}$ and $f_{2}$ are the natural frequency of the structure, the natural frequency of the support, the modal natural frequency of the first mode and the modal natural frequency of the second mode, respectively. In addition, $\omega_{c}, \omega_{p}, \gamma, \zeta_{c}$ and $\zeta_{p}$ are defined by the following equations: 


$$
\begin{aligned}
& \omega_{c}=2 \pi f_{c}=\sqrt{\frac{k_{c}}{m_{c}}} \\
& \omega_{p}=2 \pi f_{p}=\sqrt{\frac{k_{p}}{m_{p}}} \\
& \gamma=\frac{m_{c}}{m_{p}} \\
& \zeta_{c}=\frac{c_{c}}{2 \sqrt{m_{c} k_{c}}} \\
& \zeta_{p}=\frac{c_{p}}{2 \sqrt{m_{p} k_{p}}}
\end{aligned}
$$

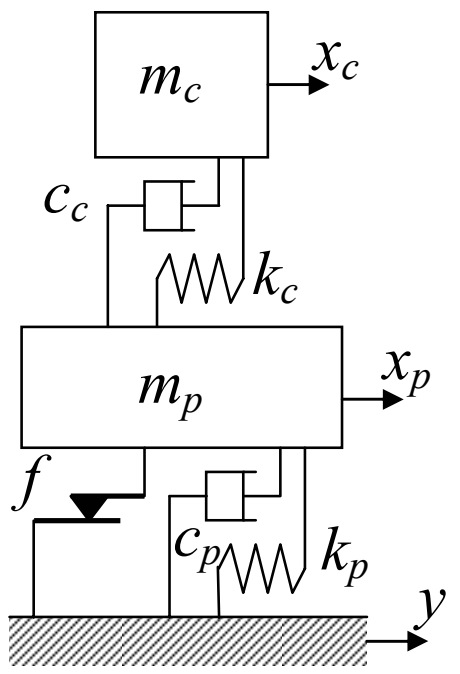

Fig.1 Two-degree-of-freedom system with friction

where $\gamma, \zeta_{c}$ and $\zeta_{p}$ are the mass ratio, the damping ratio of the structure and the damping ratio of the support, respectively. The equation is then converted to the modal space as follows:

$$
\left\{\begin{array}{l}
z_{c} \\
z_{p}
\end{array}\right\}=\left[\begin{array}{ll}
\phi_{11} & \phi_{12} \\
\phi_{21} & \phi_{22}
\end{array}\right]\left\{\begin{array}{l}
q_{1} \\
q_{2}
\end{array}\right\}=[\phi]\left\{\begin{array}{l}
q_{1} \\
q_{2}
\end{array}\right\}
$$

The equation of the 2-dof friction system in modal space is as follows:

$$
\begin{gathered}
{[\phi]^{T}[M][\phi]\left\{\begin{array}{l}
\ddot{q}_{1} \\
\ddot{q}_{2}
\end{array}\right\}+[\phi]^{T}[C][\phi]\left\{\begin{array}{l}
\dot{q}_{1} \\
\dot{q}_{2}
\end{array}\right\}+[\phi]^{T}[K][\phi]\left\{\begin{array}{l}
q_{1} \\
q_{2}
\end{array}\right\}=-[\phi]^{T}[M]\{e\} \ddot{y}+[\phi]^{T}\{f\}} \\
{[M]=\left[\begin{array}{cc}
m_{c} & 0 \\
0 & m_{p}
\end{array}\right],[C]=\left[\begin{array}{cc}
c_{c} & -c_{c} \\
-c_{c} & c_{c}+c_{p}
\end{array}\right],[K]=\left[\begin{array}{cc}
k_{c} & -k_{c} \\
-k_{c} & k_{c}+k_{p}
\end{array}\right],} \\
\{e\}=\left\{\begin{array}{l}
1 \\
1
\end{array}\right\},\{f\}=\left\{\begin{array}{l}
0 \\
-f \operatorname{sign}\left(\phi_{21} \dot{q}_{1}+\phi_{22} \dot{q}_{2}\right)
\end{array}\right\}
\end{gathered}
$$

where $\{e\}$ is the excitation vector and $\{f\}$ is the vector of the friction force. $[\phi]^{T}[M][\phi]$ and $[\phi]^{T}[K][\phi]$ become diagonal matrices. However, $[\phi]^{T}[C][\phi]$ does not become a diagonal matrix, and the friction force exists in both modes in the vector of the friction force, which is given by the following equation:

$$
[\phi]^{T}\{f\}=\left[\begin{array}{cc}
\phi_{11} & \phi_{21} \\
\phi_{12} & \phi_{22}
\end{array}\right]\left\{\begin{array}{c}
0 \\
-f \operatorname{sign}\left(\phi_{21} \dot{q}_{1}+\phi_{22} \dot{q}_{2}\right)
\end{array}\right\}=\left\{\begin{array}{c}
-\phi_{21} f \operatorname{sign}\left(\phi_{21} \dot{q}_{1}+\phi_{22} \dot{q}_{2}\right) \\
-\phi_{22} f \operatorname{sign}\left(\phi_{21} \dot{q}_{1}+\phi_{22} \dot{q}_{2}\right)
\end{array}\right\}
$$

Therefore, equation (11) is not a separated system.

The authors proposed a method of estimating the modal damping ratio without eigenvalue analysis, which can be classified as classic damping and the non-classic damping systems ${ }^{(11)(12)}$. The modal damping matrix is as follows:

$$
\left[\begin{array}{cc}
c_{1} & 0 \\
0 & c_{2}
\end{array}\right]=[\phi]^{T}\left[\begin{array}{cc}
c_{c} & -c_{c} \\
-c_{c} & c_{c}+c_{p}
\end{array}\right][\phi]
$$

where $c_{1}$ and $c_{2}$ are the damping coefficients of first and second modes, respectively. When 
the classic damping system is assumed, the following equation is obtained by equation (13):

$$
f_{1}^{2} f_{2}^{2}\left(c_{c}+c_{p}\right)-c_{p} f_{c}^{2}\left(f_{1}^{2}+f_{2}^{2}\right)+c_{p} f_{c}^{4}=0
$$

The modal natural frequencies $f_{1}$ and $f_{2}$ are as follows when $c_{1}=0$ and $c_{2}=0$ :

$$
\begin{aligned}
& f_{1}=\frac{\sqrt{2} f_{p} f_{c}}{\sqrt{(1+\lambda)\left((\gamma+1) f_{c}^{2}+f_{p}^{2}\right)}} \\
& f_{2}=\frac{\sqrt{2} f_{p} f_{c}}{\sqrt{(1-\lambda)\left((\gamma+1) f_{c}^{2}+f_{p}^{2}\right)}} \\
& \lambda=\sqrt{1-\left(\frac{2 f_{p} f_{c}}{(\gamma+1) f_{c}^{2}+f_{p}^{2}}\right)^{2}}
\end{aligned}
$$

The following equations are then obtained:

$$
\begin{aligned}
& f_{1}^{2} f_{2}^{2}=f_{c}^{2} f_{p}^{2} \\
& f_{1}^{2}+f_{2}^{2}=(\gamma+1) f_{c}^{2}+f_{p}^{2}
\end{aligned}
$$

When equations (18) and (19) are substituted for equation (14), the following equations are obtained:

$$
\begin{aligned}
& \frac{f_{c}}{f_{p}}=\frac{\zeta_{c}}{\zeta_{p}} \\
& \zeta_{1 \text { (classic) }}=\frac{\sqrt{2} \zeta_{p} \zeta_{c}}{\sqrt{(1+\eta)\left((\gamma+1) \zeta_{c}^{2}+\zeta_{p}^{2}\right)}} \\
& \zeta_{2 \text { (classic) }}=\frac{\sqrt{2} \zeta_{p} \zeta_{c}}{\sqrt{(1-\eta)\left((\gamma+1) \zeta_{c}^{2}+\zeta_{p}^{2}\right)}} \\
& \eta=\sqrt{1-\left(\frac{2 \zeta_{p} \zeta_{c}}{(\gamma+1) \zeta_{c}^{2}+\zeta_{p}^{2}}\right)^{2}}
\end{aligned}
$$

In the case of the non-classic damping system, when the left-hand and the right-hand side of equation (20) are not equal, the modal damping ratios of the first and the second modes are as follows ${ }^{(12)}$ :

$$
\begin{aligned}
& \zeta_{1}=\frac{\zeta_{p}}{\sqrt{2} \eta \sqrt{(1+\eta)\left(1+(1+\gamma) R_{f}{ }^{2}\right)^{3}}}\left[\left\{\eta+1+(\eta-1)(1+\gamma)\left(1+R_{f}{ }^{2}\right)\right\} R_{Z}+\left\{\eta-1+(\eta+1)(1+\gamma) R_{f}{ }^{2}\right\} R_{f}\right] \\
& \zeta_{2}=\frac{\zeta_{p}}{\sqrt{2} \eta \sqrt{(1-\eta)\left(1+(1+\gamma) R_{f}{ }^{2}\right)^{3}}}\left[\left\{\eta-1+(\eta+1)(1+\gamma)\left(1+R_{f}{ }^{2}\right)\right\} R_{Z}+\left\{\eta+1+(\eta-1)(1+\gamma) R_{f}{ }^{2}\right\} R_{f}\right]
\end{aligned}
$$




$$
\eta=\sqrt{1-\left(\frac{2 R_{f}}{1+(1+\gamma) R_{f}{ }^{2}}\right)^{2}}
$$

where $R_{f}\left(=f_{c} / f_{p}\right)$ is the ratio of the natural frequency and $R_{z}\left(=\zeta_{c} / \zeta_{p}\right)$ is the ratio of the damping ratio.

On the other hand, the uncoupling method of the vector of the friction force has not yet been proposed. The present study demonstrates that $\phi_{21}\left(=f_{1}^{2}-f_{c}^{2}\right)$ and $\phi_{22}\left(=f_{2}^{2}-f_{c}^{2}\right)$ in the modal matrix are functions of $\gamma$. Figure 2 shows the values of $\phi_{21}$ and $\phi_{22}$ with respect to $\gamma$ for the case of $f_{p}=1[\mathrm{~Hz}], \zeta_{p}=0.05$ and $R_{f}=R_{z}=2$ (classic damping). When $\gamma$ is nearly zero, $\phi_{22}$ becomes approximately zero. Moreover, either the friction force of the first mode or the second mode becomes approximately zero, and the direction of the friction force in the signum function becomes a simple vector. When $\phi_{22}$ is nearly zero, for example, the uncoupling of equation (12) is performed as follows:

$$
[\phi]^{T}\{f\} \fallingdotseq\left\{\begin{array}{c}
-\phi_{21} f \operatorname{sign}\left(\phi_{21} \dot{q}_{1}\right) \\
0
\end{array}\right\}
$$

Using this method, the friction force in modal space acts only in the first mode. Hence, equation (11) has been decoupled.

When the mass ratio is increased, however, the analytical error of this method is expected to increase. Therefore, the modal ratio of $\left|\phi_{21}\right|$ and $\left|\phi_{22}\right|$ can be used for estimating this error. In the present paper, the modal ratio $R_{f a i}$ is defined as follows:

$$
R_{f a i}= \begin{cases}\frac{\left|\phi_{22}\right|}{\left|\phi_{21}\right|} & \left(\left|\phi_{21}\right|>\left|\phi_{22}\right|\right) \\ \frac{\phi_{21} \mid}{\left|\phi_{22}\right|} & \left(\left|\phi_{21}\right| \leq\left|\phi_{22}\right|\right)\end{cases}
$$

Figure 3 shows the modal ratio $R_{f a i}$ in ranges of $R_{f} \leqq 3$ and $\gamma \leqq 1$. When $R_{f a i}$ closes to 1 , the accuracy of the analysis of the proposed method is reduced.

We then attempt to express the equation of the curve $R_{f a i}=1$. When $R_{f a i}$ is 1 , the values of $\left|\phi_{21}\right|$ and $\left|\phi_{22}\right|$ are equal. Therefore, equation (29) is obtained by equation (4).

$$
\left|f_{1}^{2}-f_{c}^{2}\right|=\left|f_{2}^{2}-f_{c}^{2}\right|
$$

By equations (15) and (16), the following equation is obtained:

$$
(\gamma+1) f_{c}^{2}+f_{p}^{2}=2 f_{c}^{2}
$$

Using $R_{f}=f_{c} / f_{p}$, equation (30) is expressed as follows:

$$
(\gamma+1) R_{f}^{2}+1=2 R_{f}^{2}
$$

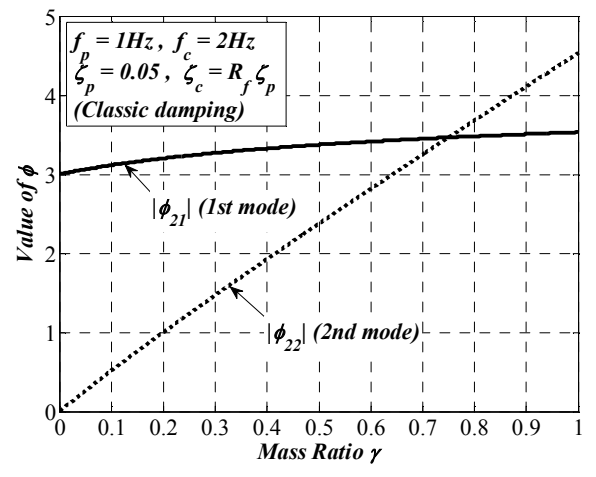

Fig.2 Values of $\left|\phi_{21}\right|$ and $\left|\phi_{22}\right|$

Hence, the line $R_{f a i}=1$ is given by the following equation: 


$$
R_{f}=\frac{1}{\sqrt{1-\gamma}}
$$

Figure 4 shows the curve of $R_{f a i}=1$. When $R_{f}$ is close to the value given by equation (32), the analytical error of this method is expected to increase.

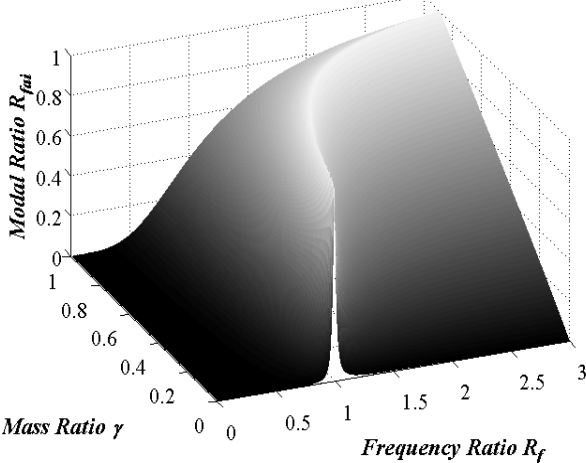

Fig.3 Modal ratio $R_{f a i}$

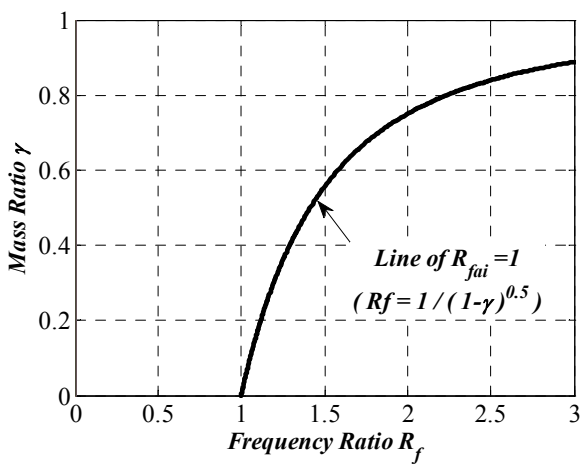

Fig.4 Curve of $R_{f a i}=1$

\section{The range of application of the modal analysis for the friction system}

The proposed method can convert dynamic equations of a 2-dof friction system into modal space. However, the modal ratio $R_{f a i}$ is changed by the mass ratio of the structure and the support. Therefore, the method of modal analysis for a 2-dof friction system has the range of application of the mass ratio or the frequency ratio. To decide this range, the response acceleration of the friction modal analysis is compared with that of 2-dof non-linear time history analysis.

\subsection{Analysis conditions}

Equation (3) divided by $m_{c}$ and $m_{p}$ is as follows:

$\left[\begin{array}{ll}1 & 0 \\ 0 & 1\end{array}\right]\left\{\begin{array}{l}\ddot{z}_{c} \\ \ddot{z}_{p}\end{array}\right\}+\left[\begin{array}{cc}2 \zeta_{c} \omega_{c} & -2 \zeta_{c} \omega_{c} \\ -2 \gamma \zeta_{c} \omega_{c} & 2 \gamma \zeta_{c} \omega_{c}+2 \zeta_{p} \omega_{p}\end{array}\right]\left\{\begin{array}{c}\dot{z}_{c} \\ \dot{z}_{p}\end{array}\right\}+\left[\begin{array}{cc}\omega_{c}^{2} & -\omega_{c}^{2} \\ -\gamma \omega_{c}^{2} & \gamma \omega_{c}^{2}+\omega_{p}^{2}\end{array}\right]\left\{\begin{array}{l}z_{c} \\ z_{p}\end{array}\right\}=-\left\{\begin{array}{l}1 \\ 1\end{array}\right\} \ddot{y}-\left\{\begin{array}{l}0 \\ \frac{f}{m_{p}} \operatorname{sign}\left(\dot{z}_{p}\right)\end{array}\right\}$

The present study defines the ratio of the friction force and the inertia force as the friction parameter $\alpha$, which is given as follows:

$$
\begin{aligned}
& \alpha=\frac{f}{A m_{p}(1+\gamma)} \\
& \begin{cases}\alpha=0 & : \text { Linear } \text { system } \\
0<\alpha<1 & : \text { Friction system } \\
\alpha \geqq 1 & : \text { Linear } \text { system }\end{cases}
\end{aligned}
$$

where $A$ is the maximum value of input acceleration. Then, using this parameter, equation (33) is transformed to the following: 


$$
\begin{array}{r}
{\left[\begin{array}{ll}
1 & 0 \\
0 & 1
\end{array}\right]\left\{\begin{array}{l}
\ddot{z}_{c} \\
\ddot{z}_{p}
\end{array}\right\}+\left[\begin{array}{cc}
2 \zeta_{c} \omega_{c} & -2 \zeta_{c} \omega_{c} \\
-2 \gamma \zeta_{c} \omega_{c} & 2 \gamma \zeta_{c} \omega_{c}+2 \zeta_{p} \omega_{p}
\end{array}\right]\left\{\begin{array}{l}
\dot{z}_{c} \\
\dot{z}_{p}
\end{array}\right\}+\left[\begin{array}{cc}
\omega_{c}^{2} & -\omega_{c}^{2} \\
-\gamma \omega_{c}^{2} & \gamma \omega_{c}^{2}+\omega_{p}{ }^{2}
\end{array}\right]\left\{\begin{array}{l}
z_{c} \\
z_{p}
\end{array}\right\}} \\
=-\left\{\begin{array}{l}
1 \\
1
\end{array}\right\} \ddot{y}-\left\{\begin{array}{l}
0 \\
A \alpha(1+\gamma) \operatorname{sign}\left(\dot{z}_{p}\right)
\end{array}\right\}
\end{array}
$$

The result of 2-dof non-linear time history analysis is obtained by equation (36), and the result of modal analysis for the 2-dof friction system is obtained by equations (10) and (11). The present study adopts the Runge-Kutta-Gill method for numerical integration. The condition of the analysis is shown in Table 1. Figure 5 shows the input wave for the system. In order to estimate the error of the uncoupling method, the classic damping system is assumed $\left(R_{f}=R_{z}\right)$.

Table 1. Analysis conditions

\begin{tabular}{|c|c|}
\hline Natural frequency of support $f_{p}[\mathrm{~Hz}]$ & 1 \\
\hline Frequency ratio $R_{f}$ & $0.01 \sim 3$ \\
\hline Damping ratio of support $\zeta_{p}$ & 0.05 \\
\hline Ratio of damping ratio $R_{z}$ & $R_{z}=R_{f}$ \\
\hline Mass ratio $\gamma$ & $0.001 \sim 0.1$ \\
\hline Friction force $\alpha$ & $0.1 \sim 0.5$ \\
\hline Input wave & Sinusoidal, $1[\mathrm{~Hz}]$ \\
\hline Duration time [sec] & 30 \\
\hline Sampling time [sec] & 0.001 \\
\hline
\end{tabular}

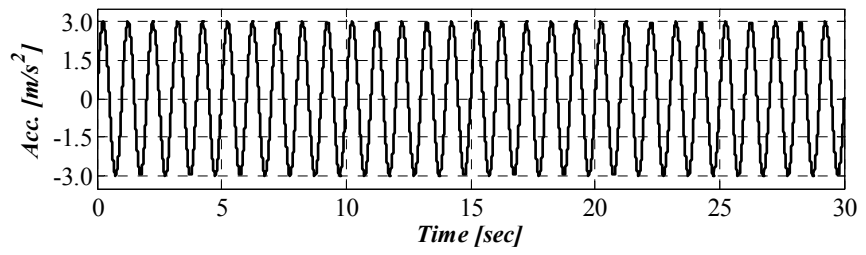

Fig.5 Input wave (sine wave, $1[\mathrm{~Hz}]$ )

\subsection{Error of modal analysis method for the friction system}

Figure 6 shows a comparison of the time history of response acceleration obtained by two methods in the case of $f_{p}=1[\mathrm{~Hz}], R_{f}=1, \gamma=0.01$ and $\alpha=0.3$. Figure 6(a) shows the response accelerations at the structure, and Fig. 6(b) shows that at the support.

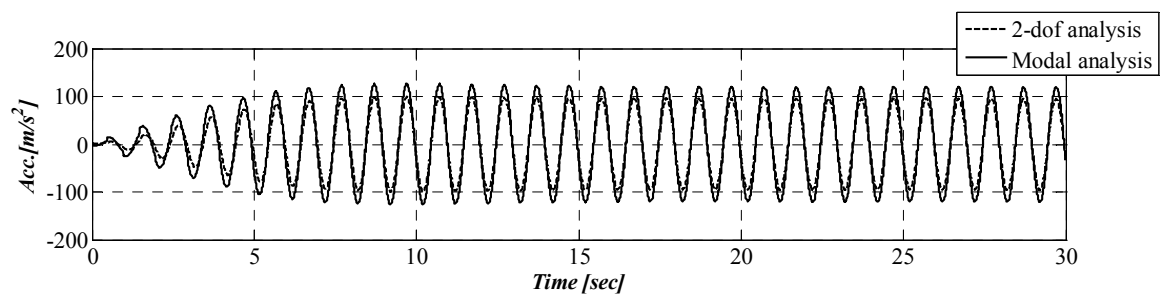

(a) Structure

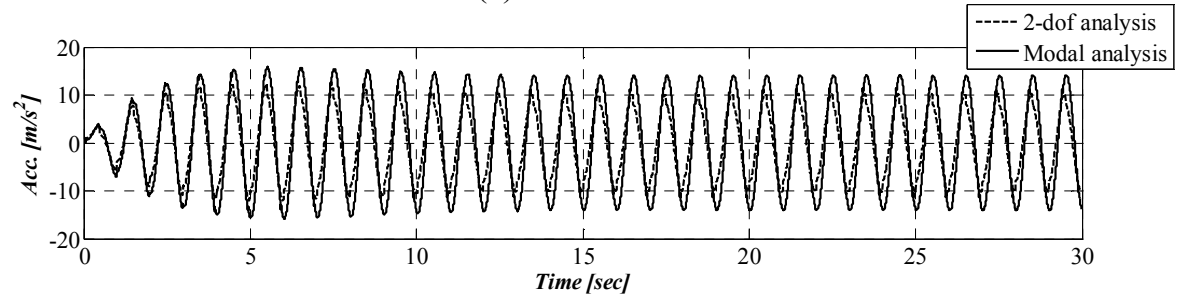

(b) Support

Fig.6 Time history of acceleration $\left(f_{p}=1[\mathrm{~Hz}], R_{f}=1, \gamma=0.01, \alpha=0.3\right)$ 
Figure 6 shows the analytical error observed by the proposed method for the 2-dof friction system. In this analysis, the error of the proposed method depends on $R_{f}, \gamma$ and $\alpha$. Therefore, the present study calculates the error in the range of Table 1 . The error of the proposed method is defined as follows:

$$
\operatorname{Error}[\%]=\left|\frac{\operatorname{Max}\left(\ddot{x}_{\text {modal }}\right)-\operatorname{Max}\left(\ddot{x}_{2 d o f}\right)}{\operatorname{Max}\left(\ddot{x}_{2 d o f}\right)}\right| \times 100
$$

where $\operatorname{Max}\left(\ddot{x}_{\text {modal }}\right)$ and $\operatorname{Max}\left(\ddot{x}_{2 \text { dof }}\right)$ are the maximum value of response acceleration obtained by the friction modal analysis and that obtained by 2-dof non-linear time history analysis, respectively. Moreover, the present study defines the area of the error under $10 \%$ as the applicable area of the modal analysis method for friction. Figures 7 through 11 show the areas of error over $10 \%$ obtained by friction modal analysis. In these figures, (a) shows the area of the error over $10 \%$ at the structure and (b) shows that at the support.

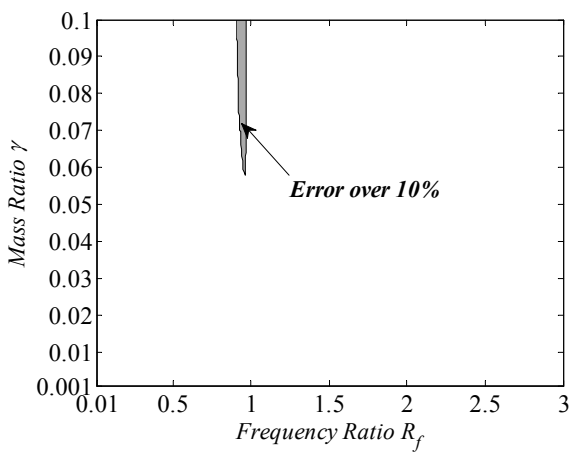

(a) Structure

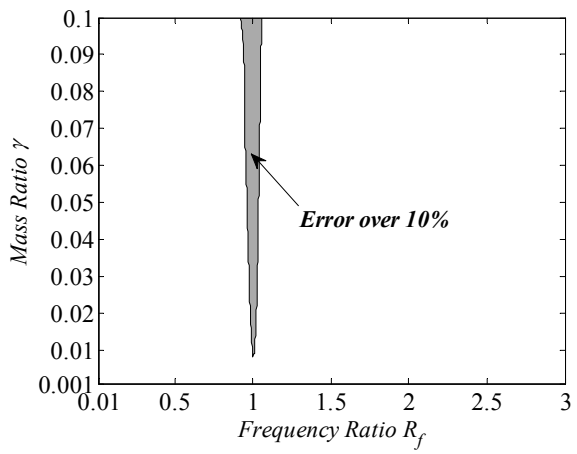

(b) Support

Fig.7 Area of error over $10 \%(\alpha=0.1)$

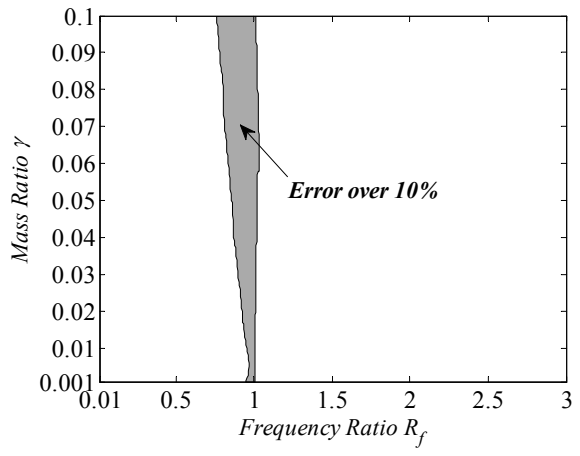

(a) Structure

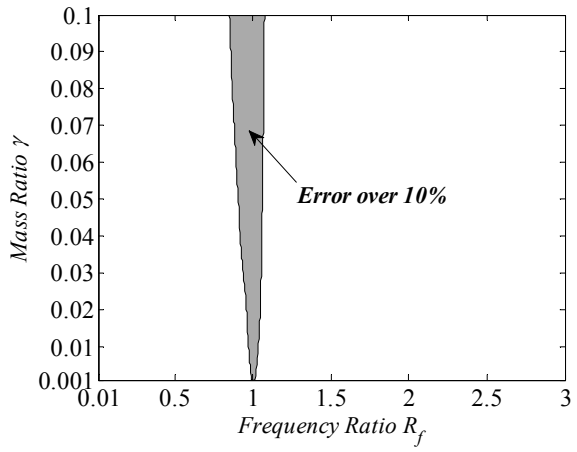

(b) Support

Fig. 8 Area of error over $10 \%(\alpha=0.2)$

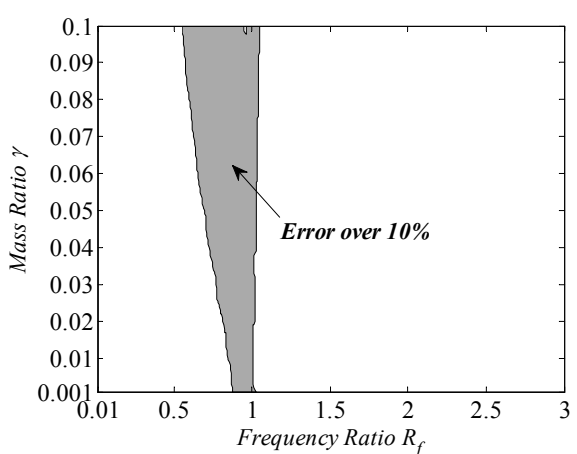

(a) Structure

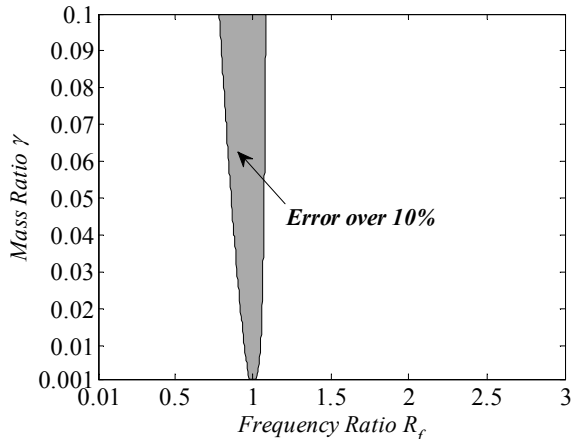

(b) Support

Fig.9 Area of error over $10 \%(\alpha=0.3)$ 


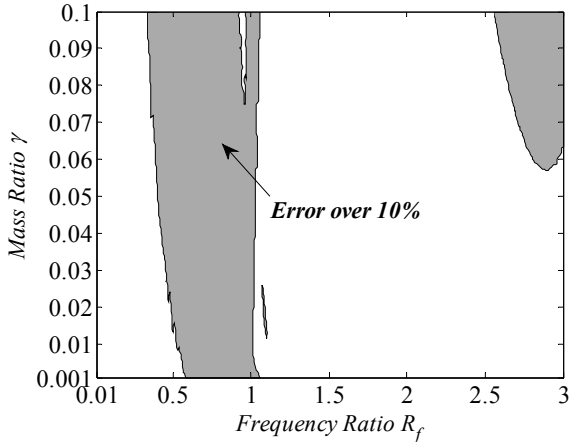

(a) Structure

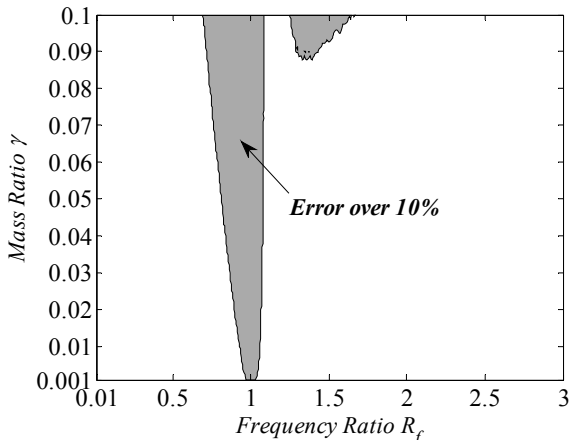

(b) Support

Fig.10 Area of error over $10 \%(\alpha=0.4)$

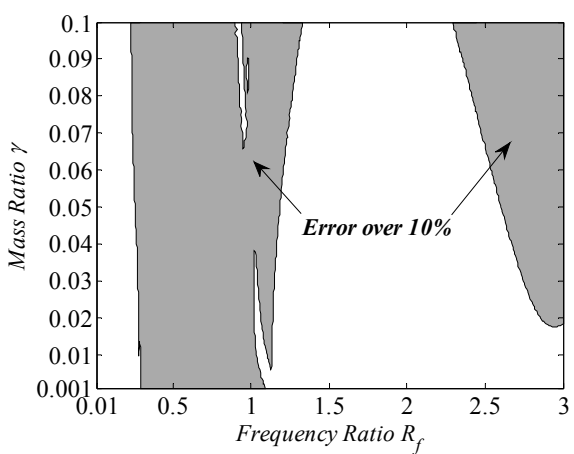

(a) Structure

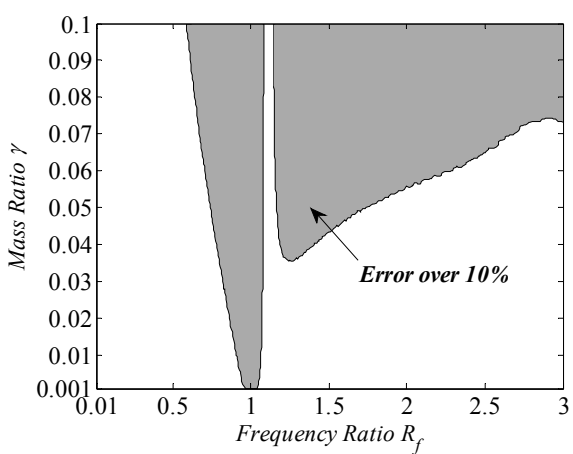

(b) Support

Fig. 11 Area of error over $10 \%(\alpha=0.5)$

From these figures, we can judge whether the proposed method is applicable for each parameter. The applicable area becomes small with increasing $\alpha$. This is not a problem under the practical conditions, because $R_{f}$ always exceeds 1 in industrial structures.

For example, we attempt to obtain the range of application of modal analysis for 2-dof friction system, which is $f_{p}=1[\mathrm{~Hz}]$ and $\gamma=0.01$. The maximum acceleration of the input wave is $3\left[\mathrm{~m} / \mathrm{s}^{2}\right]$. The materials of the sliding plane are iron and PTFE. The friction coefficient $\mu$ between iron and PTFE is 0.10 , and the friction force is defined as follows:

$$
\mu N_{p}=\mu\left(m_{p}+m_{c}\right) g=\mu(1+\gamma) m_{p} g
$$

The friction force $\alpha$ is then calculated as follows:

$$
\begin{aligned}
\alpha & =\frac{f}{A m_{p}(1+\gamma)}=\frac{\mu m_{p}(1+\gamma) g}{A m_{p}(1+\gamma)}=\frac{\mu g}{A} \\
& =\frac{0.1 * 9.8}{3}=0.326 \cdots \fallingdotseq 0.3
\end{aligned}
$$

From Fig. 9, the range of application for the structure is $R_{f} \leqq 0.84$ and $1.01 \leqq R_{f}$, and that for the support is $R_{f} \leqq 0.94$ and $1.05 \leqq R_{f}$. Hence, the range of application is obtained as $R_{f} \leqq 0.84$ and $1.05 \leqq R_{f}$. 


\section{Conclusion}

In the present study, the method of modal separation for a 2-dof friction system using the modal ratio $R_{f a i}$ of $\left|\phi_{21}\right|$ and $\left|\phi_{22}\right|$ in the modal matrix is considered. One mode of the vector of the friction force can be regarded as approximately zero when the mass ratio $\gamma$ is nearly zero. The proposed approximation method can convert dynamic equations of a 2-dof friction system into modal space. Analytical errors of the maximum acceleration at the structure and support obtained by the proposed method are calculated and compared with the 2-dof non-linear time history analysis. This error varies with the modal ratio $R_{f a i}$ with regard to $\gamma$. The formula $R_{f}=1 / \sqrt{1-\gamma}$ can expect the condition that the maximum error would happen. The results of the present study revealed that an area of error of over $10 \%$ for the modal analysis method for a 2-dof friction system. Therefore, the range of application of $R_{f}$ and $\gamma$ can be easily estimated using this error area.

\section{References}

(1) Marui, E., Kato, S., Forced vibration with solid friction, Trans. of JSME, Series C, Vol.49 No.443(1983), pp.1146-1152.

(2) Watanabe, T., et al, Vibration Test of Plant Piping System Having Friction Support, Trans. of JSME, Series C, Vol.58, No.552(1992), pp.17-23.

(3) Watanabe, T., et al, Seismic Responses of Plant Piping System Having Friction an Vibro-impact Characteristics, Trans. of JSME, Series C, Vol.62 No.595(1996), pp.63-68.

(4) Watanabe, T., Suzuki, K., Seismic Response of Piping System with Friction Sliding on a Plane, Trans. of JSME, Series C, Vol.64 No.617(1998), pp.64-69.

(5) Watanabe, T., Suzuki, K., Estimation of Equivalent Damping Ratio using Friction Response Spectrum, Trans. of JSME, Series C, Vol.66 No.642(2000), pp.87-92.

(6) Tomoda, A., Watanabe, T., Tanaka, K., Study on Friction Response Spectrum Under Long Period System, Trans. of JSME, Series C, Vol.73 No.730(2007), pp.1699-1704.

(7) Aoki, S., et al, Computer Aided Method of Analysis for Vibration, Corona publishing co., pp.233-240.

(8) Watanabe, T., Suzuki, K., Seismic Response Analysis of Piping System with Friction Support (Maximum Response Estimation Using Frictional Response Spectrum), Trans. of JSME, Series C, Vol.64 No.619(1998), pp.87-92.

(9) Hanawa, Y., et al, Vibration Test and Analysis of Response of Piping System with Friction Support, Trans. of JSME, Series C, Vol.64 No.640(1999), pp.4611-4617.

(10) Nagamatsu, A., Modal Analysis, Baifukan, pp.53-61.

(11) Watanabe, T., Tomoda, A., Tanaka, K., Estimation of the Damping Ratio of the Coupled System Based on the Damping Ratios of Two 1-Dof Systems, Journal of System Design and Dynamics, Vol. 2, No. 4 (2008), pp.1006-1014.

(12) Watanabe, T., Tomoda, A., Tanaka, K., Estimation of the Damping Ratio of the Coupled System Based on the Damping Ratios of Two 1-Dof Systems, Trans. of JSME, Series C, Vol.74 No.748 (2008), pp.2886-2893. 